\title{
Real-Time Tree Foliage Estimation Using a Ground Laser Scanner
}

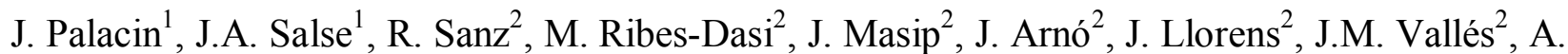 \\ Escolà $^{2}$, P. Massana ${ }^{2}$, F. Camp ${ }^{2}$, F. Solanelles ${ }^{2}$, J.R. Rosell ${ }^{2}$ \\ ${ }^{1}$ Department of Informatics and Industrial Engineering, University of Lleida \\ ${ }^{2}$ Department of Agro-forestry Engineering, University of Lleida \\ Jaume II, 69, 25001 Lleida, Spain \\ Phone: +34-973-702700, Fax: +34-973-702702, Email: palacin@ieee.org
}

\begin{abstract}
The optimization of most pesticide and fertilizer applications is based on overall grove conditions. In this work we propose a measurement system based on a ground laser scanner to estimate the volume of the trees and then extrapolate their foliage surface in real-time. Tests with pear trees demonstrated that the relation between the volume and the foliage can be interpreted as linear with a coefficient of correlation $(R)$ of 0.81 and the foliar surface can be estimated with an average error less than $5 \%$.
\end{abstract}

Keywords - LIDAR, laser ground scanner, agrochemical dose control.

\section{INTRODUCTION}

Traditional pesticide and fertilizer application in grove treatment are based on uniform application causing significant material loss (fig. 1). The measurement of the overall grove conditions can be used to optimize the application, providing economic benefits for growers and reducing pollution in the environment. The measurement of tree canopy structural characteristics, such as volume and foliage can be carried out by means of several detection principles, such as image analysis techniques, stereoscopy photography, analysis of the light spectrum, infrared thermograph, ultrasonic sensors, and light detection and ranging (LIDAR) laser sensors.

However, image based canopy measurement requires elaborated algorithms and huge computational resources to operate in real-time. Ultrasonic sensors have been used to measure canopy volume for pesticide applications [1, 2, 3, 4] although, the divergence angle of the ultrasonic waves limits the spatial resolution and the accuracy of the measurements.

LIDAR is a remote sensing technique based on the measurement of the time a laser pulse takes between the sensor and a target and has the advantage that the beam can be very thin and diverges very little. In recent years, the LIDAR has been widely used for the measurement of environmental target parameters in forest and agricultural characterization. The canopy height [5] and tree height and volume [6] were estimated using an airborne LIDAR altimeter. Walkate [7] used the LIDAR to study the relationship between orchard tree crop structure and performance characteristics of an axial fan sprayer with good correlation between spray deposit and some measure of the foliage density. Tumbo [3] compared the performance of ultrasonic and laser sensors for measurement of citrus canopy volume obtaining good correlations with manual measurements. Recently, Wei $[9,10]$ used a terrestrial LIDAR to measure tree height, width and volume developing a set of experiments to evaluate the repeatability and accuracy of the measurements, obtaining a coefficient of variation of $5.4 \%$ and a relative error of $4.4 \%$ in the estimation of the volume but without real-time capabilities. These experiments were partially reproduced in [11] by the authors.

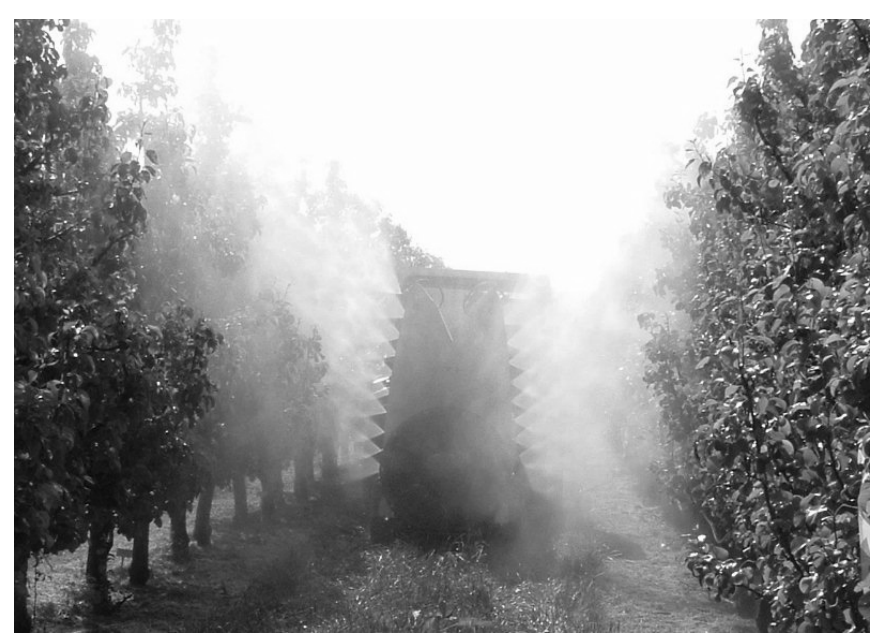

Fig. 1. Pesticide application.

The pesticide and fertilizer application can be optimized in two ways: adjusting the overall dose to guarantee uniform application, or having an estimate of the foliar surface of the surrounding trees to adequate the application dose. Normally, uniform application requires an estimate of the real displacement of the application unit and adjusting the overall dose. However, in the practice, the uniform dose is accomplished fixing a dose and an uniform speed in the tractor unit for the entire grove because the tractor has power enough to guarantee a certain degree of uniformity in the speed in all the application even when using a very simple speed control loop. This work is focused on the second alternative: the development of a measurement system for real-time foliage estimation that could be incorporated to any kind of fertilizer or pesticide machine with dose control capabilities to adjust the quantity of applied product according to the crop characteristics. 
The main hypothesis of this work is that there is a relation between the volume and the foliar surface of the trees. With this relation, a measurement of the volume of the trees could be used to estimate their foliar surface and then optimize pesticide and fertilizer application. The fertilizer is usually applied directly to the ground and only an overall estimation of the entire surrounding foliar surface is enough to control the application. The pesticide is usually applied directly to the trees (fig. 1) using the same dose for the entire tree and grove but application machines normally have the capability to change the dose depending on the height. Therefore, it could be very useful to have an estimation of the foliar surface at different heights. In this work the pear trees grove is the case example and the estimation of the foliar surface and the realtime capabilities are the main improvement related to the previous work.

This paper will be structured as follows; In Section 2 we will describe our approach; In Section 3 we will describe the experimental results obtained; In section 4 we will discuss the results obtained and some improvements. The paper ends with the main conclusions and advantages over similar works.

\section{MATERIALS AND METHODS}

The method to obtain an estimation of the foliar surface is based on the estimation of the volume of the trees.

\section{A. Tree volume measurement}

Tree volume measurement is obtained using a two dimensional LIDAR measurement system [9, 10]. Figure 2 shows a LIDAR attached to a tractor unit and figure 3 show the schema of the proposed tree foliage measurement system composed by the LIDAR measurement system and i) a computer for data storage and off-line data processing or ii) a dedicated programmable automation controller for real-time tree foliage estimation. The LIDAR used is a low-cost general-purpose LMS-200 model (Sick, Düsseldorf, Germany) [12] (fig. 4), a fully-automatic divergent laser scanner based on the measurement of time-of-flight (TOF) $[13,14]$ with an accuracy of $\pm 15 \mathrm{~mm}$ in a single shot measurement and $5 \mathrm{~mm}$ standard deviation in a range up to 8 $\mathrm{m}$. The time between the transmission and the reception of the pulsed near-infrared laser beam is used to measure the distance between the scanner and the reflecting object surface. The laser beam is deflected by a rotating mirror turning at $4500 \mathrm{rpm}$ (75 rps) (fig. 4), which results in a fanshaped scan pattern where the maximum scanning angle is $180^{\circ}$. The angular resolution is selectable at $l^{\circ}, 0.5^{\circ}$, or $0.25^{\circ}$ making 181, 361 and 400 measures respectively at full scanning range with a response time of 13,26 and $53 \mathrm{~ms}$ respectively. The LMS-200 has a standard RS232 serial port for data transfer with a rate selectable at $9.6,19.2$ or 38.4 Kbaud and a non-standard RS422 serial port capable of 500 Kbaud using a specific RS422 card.

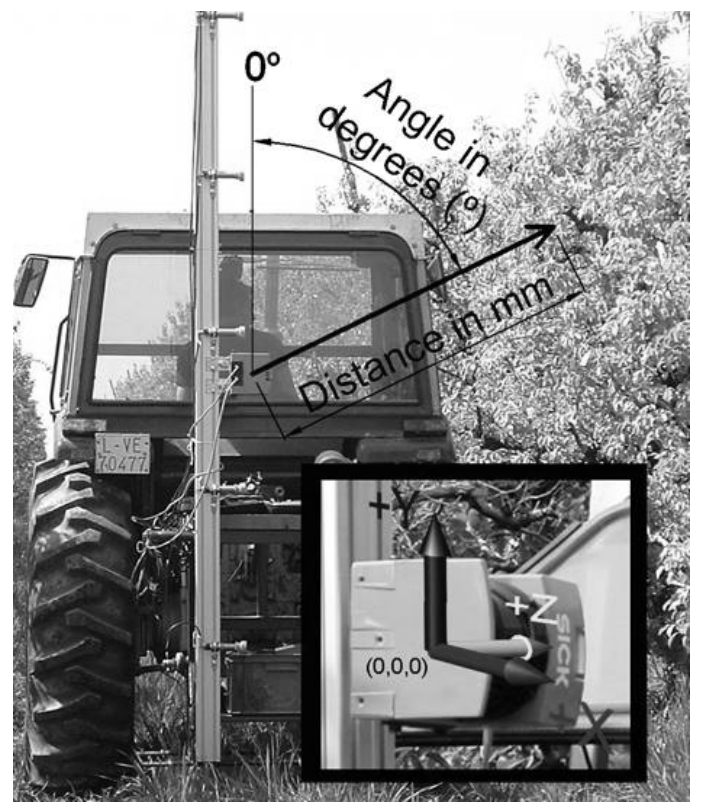

Fig. 2. LIDAR attached to a tractor unit.

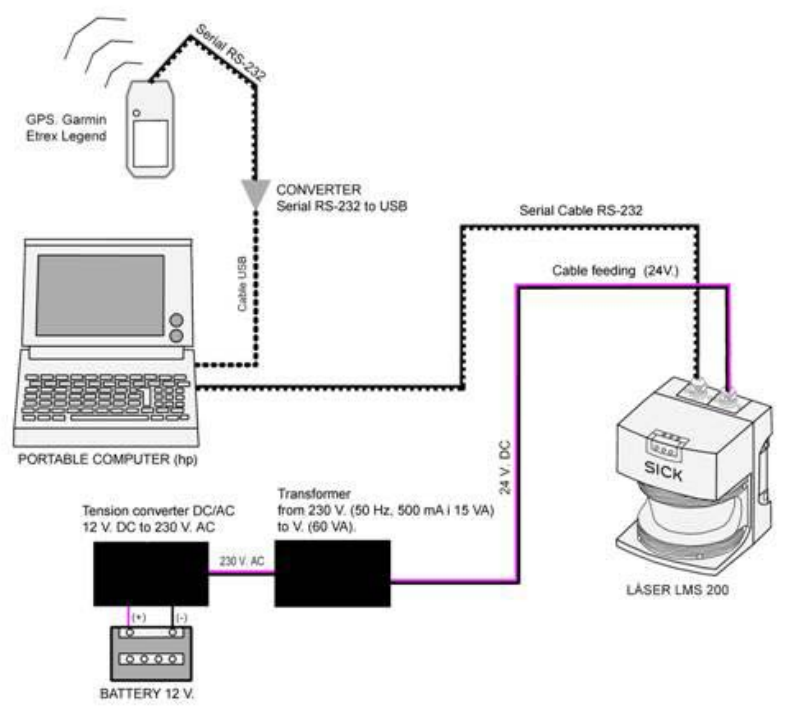

Fig. 3. Electronic equipment of the tree foliage measurement system.
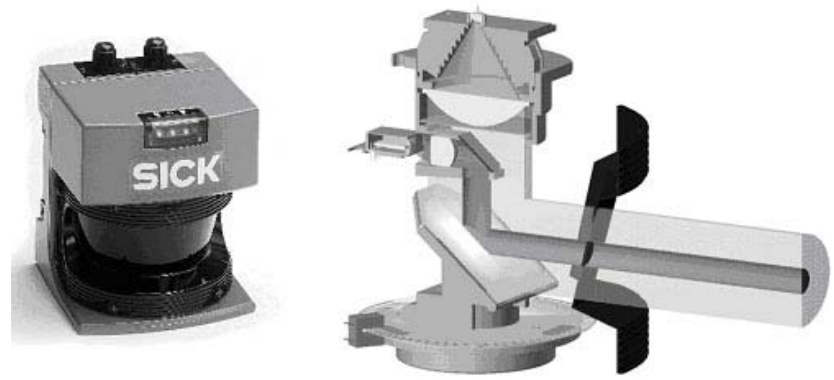

Fig. 4. Detail of the LMS-200.

The distance measured by the LMS-200 can be configured in three different modes: polar, rectangular, and reflexivity. In polar mode the supplied data are composed by a sequence 
of distances. In rectangular mode the data are composed by a pair of relative distances $[X, Y]$. In reflexivity mode the data sent are composed by a sequence of the reflexivity measured. In all the sequences the first value corresponds to the initial measurement angle (normally $0^{\circ}$ ), the second value corresponds to the next angle which depends on the angular resolution selected, and so on. Moreover, each mode can be operated in single scan (the scan is requested by the control unit) or in continuous scan (the operation is started and stopped by the control unit) where the LMS-200 operates at full speed sending each slice as generated. Additionally, the LMS-200 can be configured to average a number of consecutive scans for noise reduction and to reduce the scanning range. The slices obtained with the LIDAR are used to compute the volume of the trees.

For the proposed application the LIDAR was configured for non-averaged single-scan polar distance measurement with a range up to $8 \mathrm{~m}$ and with an angular resolution of $1^{\circ}$ in a range of $\left[0^{\circ} \ldots 180^{\circ}\right]$. The external communication were performed through the RS232 serial port at 38.4 kbaud. In the development stage the LIDAR was interfaced to a computer for data recording and a graphic user interface was developed in MatLab (The Mathworks Inc, Natick, Massachusetts) for off-line processing and algorithm development. In the final test stage the LIDAR was interfaced to a Compact FieldPoint programmable automation controller (National Instruments Corporation, Austin, Texas) for real-time operation.

\section{B. Algorithm formulation}

Figures 5 and 6 shows a top and frontal view of the scanning scenario composed by a tree row line and a LIDAR following a trajectory parallel to the trees where:

$\theta=$ angular resolution of the scans.

$j=$ beam index into the scan, starting in the vertical position.

$i=$ scan index.

FTS $=\quad$ forward travel speed of the LIDAR.

$\mathrm{D}_{\mathrm{i}}=$ distance between the LIDAR travel line and the tree row line for the scan $i$.

$\mathrm{D}_{\mathrm{T}}=$ distance between two parallel rows of trees.

$\mathrm{H}_{\mathrm{S}}=$ height of the LIDAR above ground.

$\mathrm{H}_{\mathrm{G}}=$ height of grass above ground.

$\mathrm{W}_{\mathrm{T}}=$ distance between two consecutive trees in the tree row line.

$\Delta \mathrm{W}=$ distance interval between two consecutive vertical scans.

$\Delta \mathrm{t}=$ time interval between two consecutive vertical scans.

$\beta_{\mathrm{j}}=$ angle between the vertical direction and laser beam $j$ $\left(\beta_{\mathrm{j}}=\mathrm{j} \cdot \theta\right)$.

$\mathrm{L}_{\mathrm{ij}}=$ distance measured from the LIDAR to the target for slice $i$ and beam $j$.

$\mathrm{LL}_{\mathrm{ij}}=$ distance from the LIDAR to the vertical plane that contains the tree row line.

Only $\mathrm{H}_{\mathrm{S}}, \theta$ and the structural parameters of the trees $\mathrm{D}_{\mathrm{T}}$ and $\mathrm{W}_{\mathrm{T}}$ are known a priori.

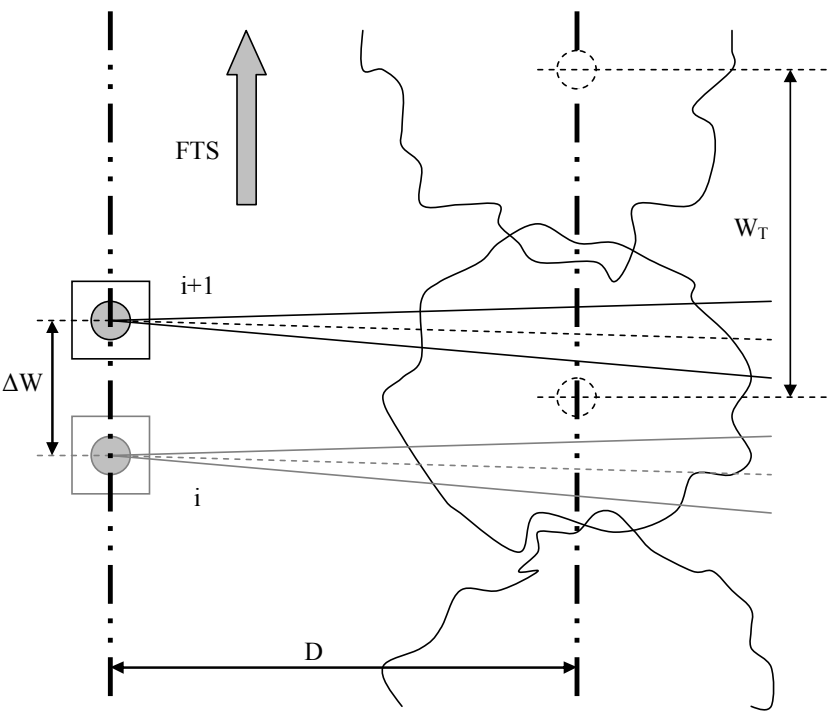

Fig. 5. Top view of the scanning scenario.

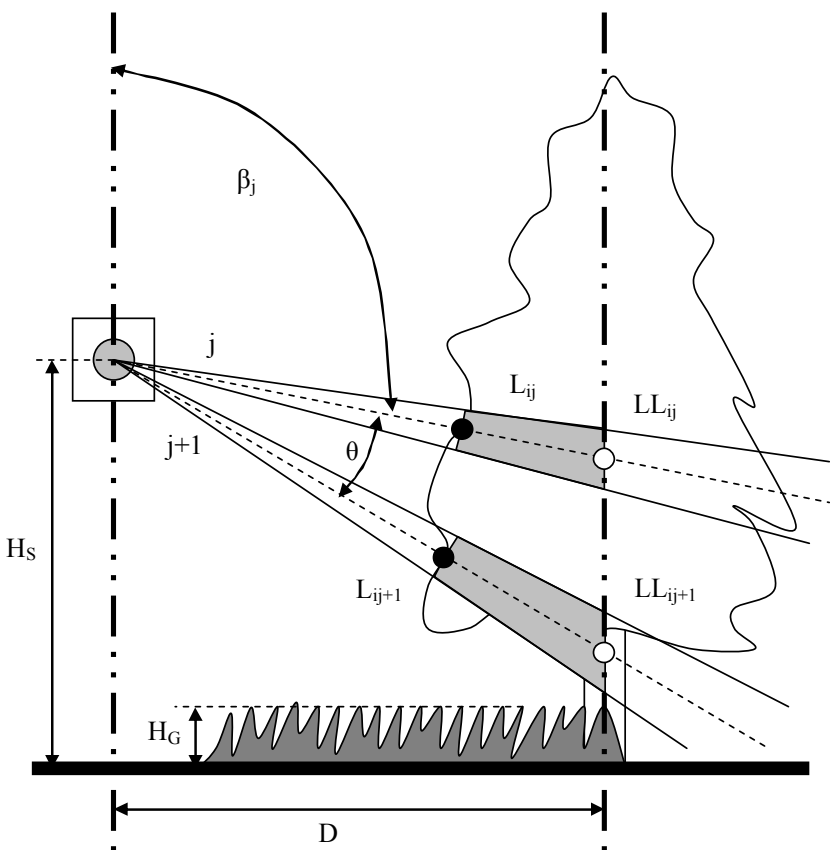

Fig. 6. Frontal view of the scanning scenario.

The final goal is to find a relation between the volume and the foliage surface; therefore both values must be estimated or measured. The volume can be estimated using the data obtained by the LIDAR but the foliage surface must be measured collecting manually the foliage in the tree and measuring the total surface using a specialized machine [15]. Obviously, this destructive measurement must be done after the LIDAR scanning and only for calibration purposes.

Moreover, a set of different boxes are defined to estimate the volume and the foliage surface at different heights. The boxes cover a number of trees because, in a normal grove, it is impossible to estimate the volume of an individual tree. Each box represents the volume and the foliar surface of one 
side of several trees. Figure 7 show an image of a pear tree crop and figure 8 shows the data obtained with the LIDAR and the boxes defined for calibration.

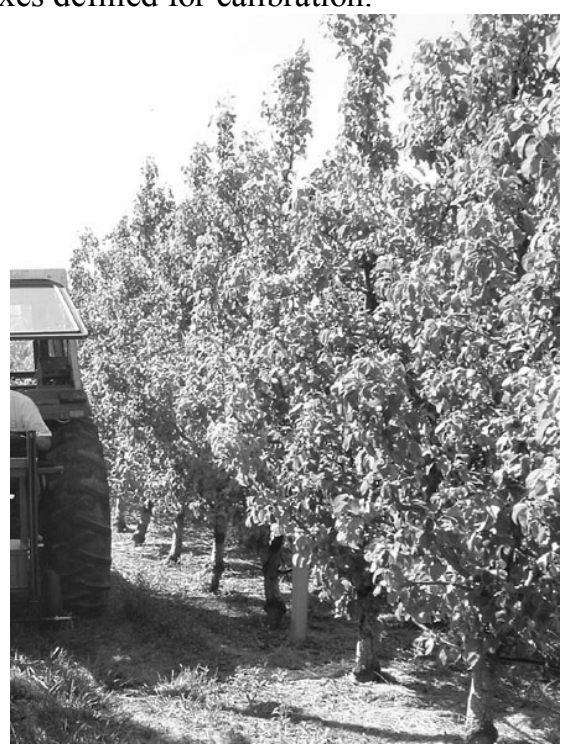

Fig. 7. Image of a typical pear tree crop.

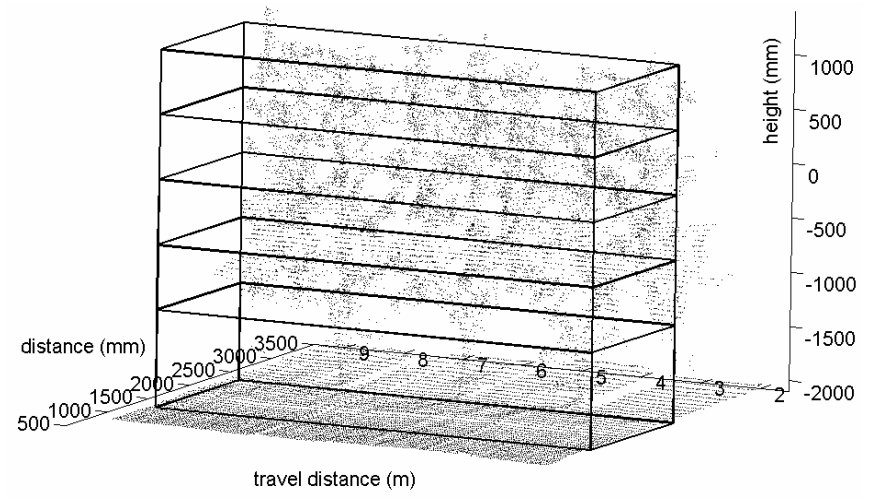

Fig. 8. LIDAR image of a typical pear tree crop obtained with $\mathrm{H}_{\mathrm{S}}=$ $2100 \mathrm{~mm}, D_{i}=1470 \mathrm{~m}, \mathrm{FTS}=0.322 \mathrm{~m} / \mathrm{s}, \Delta \mathrm{W}=31 \mathrm{~mm}$. The measurement boxes are also plotted.

The slices provided by the LIDAR can be integrated to obtain the volume, $V$, of a given area.

$$
V(k, p)=\sum_{i=k}^{p} \sum_{j=0}^{N-1} S_{i j} \cdot \Delta W,
$$

where $S_{i j}$ is the surface between the $j$ and $j+1$ laser measurement in the slice $i, k$ and $p$ are the starting and ending travel slices, $N$ the number of measurements in one slice. This surface can be computed if the distance to the vertical plane that defines the center of the tree row line, $D_{i}$, and the rectangular coordinates $\left[X_{i j}, Y_{i j}\right]$ of each measurement point, $L_{i j}$, are available. For example, if $\left(X_{i j} \leq D_{i}\right)$ and $\left(X_{i j+1} \leq D_{i}\right)$ :

$$
S_{i j}=\frac{\left(2 \cdot D_{i}-\left(X_{i j}+X_{i j+1}\right)\right)}{2} \cdot\left(Y_{i j}-Y_{i j+1}\right) .
$$

The volume of the tree and the foliar surface are obtained for each box. However, depending on the relative location of the box and the points $L_{i j}$ and $L_{i j+1}$ a set of special situations appears, all of them must be carefully considered to improve the speed of the computation of $S_{i j}$.

Finally, figure 9 compares the estimation of the volume obtained with the LIDAR and the foliar surface obtained manually. The results correspond to five different areas of the same scanned grove, in alternate days, obtained with the LIDAR and manually defoliated. In each procedure, five boxes have been defined with height of [0 900], [900 1500], [1500 2100], [2100 2700], [2700 3300] mm relative to the ground; the width is always different but at least covering 3 consecutive trees.

The relation between the volume and foliar surface shown in figure 9 can be extracted with a first (fig. 9-line) and second order least squares approximation obtaining a coefficient of correlation of 0.81 and 0.86 respectively. In this calibration, a large amount of points corresponding to different foliage areas of the grove must be used to be representative otherwise a coefficient of correlation better than 0.96 is obtained in all cases using only one set of data. However, a maximum error of $93 \%$ is obtained using a linear approximation in the estimation of the foliar surface but the average error in the estimation is only $16 \%$, small enough to allow the use of the proposed measurement system. The maximum error is mainly originated in the first level, [0 900] $\mathrm{mm}$, because in this area the foliar surface is very small but the volume measured is high (fig. 9-circle) due to the trunk of the trees.

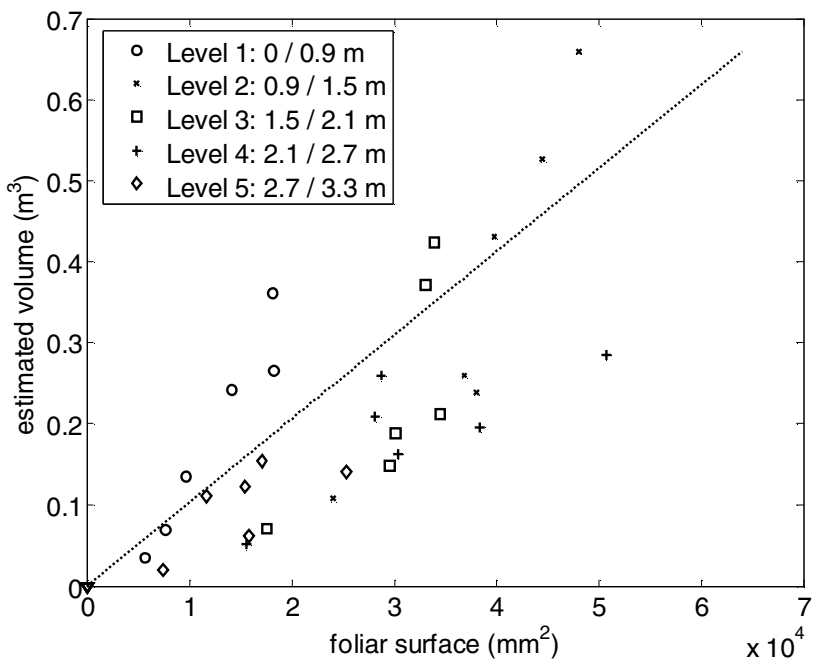

Fig. 9. Relation between volume and foliar surface for pear trees.

\section{EXPERIMENTAL RESULTS}

The experimental evaluation of the proposed measurement system is based on the estimation of the foliar surface of a part of the grove. To this end, several lines of trees were scanned using a LIDAR attached in the back of a tractor unit. However, the main drawback is that the manual defoliation of 
the trees is also needed in order to compare the results obtained. Therefore, in order to optimize the effort of the manual defoliation, the selected areas were scanned several times and with different conditions. Additionally, at this stage, other important aspects as the height of the grass and the distance from the LIDAR to the center of the trees were also controlled and fixed to avoid their influence in the measurements.

The data points of the slices obtained with the LIDAR are integrated to compute a differential volume associated with each slice. This volume is converted in foliar surface using the linear relation obtained in the previous section. In normal operation, a pesticide machine (fig. 1) is better controlled if the estimation of the foliar surface is provided for different heights. To this end, the differential volume of the slice can be computed between a set of heights defined by the user However, for an easy experimental evaluation, the heights coincide with the boxes used for defoliation.

Figure 10 shows an image of the foliar surface estimated by the measurement system for five different heights where the grey level depicts the instantaneous foliar surface estimated for each slice area. Obviously, sprayer or diffuser used for the agrochemical application does not used have the degree of resolution offered by the measurement system. Thus, a sliding window of slices must be averaged depending on the real area covered by the applicator. Finally, table I shows the relative error obtained in the estimation of the foliar surface of a line of trees, evaluated in different days and in different areas of the same grove.

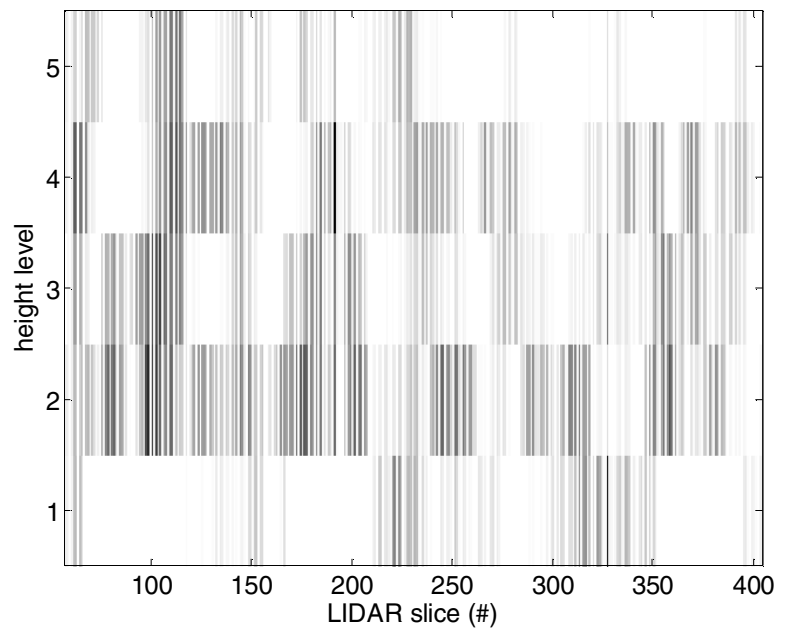

Fig. 10. Estimated absolute foliar surface of a pear tree crop obtained with $\mathrm{H}_{\mathrm{S}}=2100 \mathrm{~mm}, \mathrm{D}_{\mathrm{i}}=1470 \mathrm{~m}, \mathrm{FTS}=0.322 \mathrm{~m} / \mathrm{s}, \Delta \mathrm{W}=31 \mathrm{~mm}$.

\section{DISCUSSION}

The proposed measurement system is based on the hypothesis that all the volume of the trees can be interpreted as foliar surface. This hypothesis can be accepted for a certain varieties of species but not for the one analyzed in this work because of his long trunk. This effect can be observed in figure 9 because the data corresponding to the first height, $\left[\begin{array}{ll}0 & 0.9\end{array}\right] \mathrm{m}$, has a slope greater than the other points due to his high volume and low foliar surface. Probably, this is the cause of the poor coefficient of correlation obtained with the linear approximation, $\mathrm{R}=0.81$. The effect of the trunk is clearly observed in table I because the foliar surface is overestimated in the lower heights whereas is under-estimated in the higher heights.

The best way to avoid the effect of the trunk is to have an initial estimation of the volume of the wood of the trees, for example, with an initial scanning in winter. However, this alternative is too complex because the volume of the trees must be measured, stored and precisely geo-referenced to allow its posterior compensation, increasing the complexity and the computational power needed for a real-time measurement system. Alternatively, we propose the following approach to compensate the trunk volume: weight the estimation of the foliar surface with the average error values obtained at different height on table I. Table II shows the relative error obtained with the height-weighted estimation. The average relative error in the estimation of the foliar surface has been reduced in all heights and all have positive values with an interesting slightly over-estimation of the foliage surface obtained. Figure 11 shows the weights applied for the 5 heights used in the analysis and a second order least squares analysis to estimate the weights to apply in case of using other levels. However, although we have not tested, it is supposed that the proposed improvement is dependent on the specie and the height of the tree.

Table I. Relative error in the estimation of the foliar surface.

\begin{tabular}{|c|c|c|c|c|c|c|}
\hline $\begin{array}{c}\text { Height } \\
(\mathrm{m})\end{array}$ & Case 1 & Case 2 & Case 3 & Case 4 & Case 5 & Average \\
\hline$\left[\begin{array}{llll}2.7 & 3.3\end{array}\right]$ & $-39 \%$ & $-4.3 \%$ & $-32 \%$ & $-32 \%$ & $-14 \%$ & $-26.8 \%$ \\
\hline$\left[\begin{array}{ll}2.1 & 2.7\end{array}\right]$ & $-22 \%$ & $-25 \%$ & $-7.1 \%$ & $-23 \%$ & $-21 \%$ & $-25.7 \%$ \\
\hline$\left[\begin{array}{ll}1.5 & 2.1\end{array}\right]$ & $-5.5 \%$ & $-38 \%$ & $-12 \%$ & $5.8 \%$ & $-4.1 \%$ & $-10.4 \%$ \\
\hline$\left[\begin{array}{ll}0.9 & 1.5\end{array}\right]$ & $6.6 \%$ & $8.9 \%$ & $9.2 \%$ & $0.4 \%$ & $8.2 \%$ & $7.6 \%$ \\
\hline$\left[\begin{array}{ll}0 & 0.9\end{array}\right]$ & $48 \%$ & $72 \%$ & $55 \%$ & $22 \%$ & $93 \%$ & $59.6 \%$ \\
\hline$\left[\begin{array}{ll}0 & 3.3\end{array}\right]$ & $-4.3 \%$ & $-5.4 \%$ & $-2.6 \%$ & $-3.8 \%$ & $1.1 \%$ & $-4.8 \%$ \\
\hline
\end{tabular}

Table II. Relative error for the height-weighted estimation.

\begin{tabular}{|c|c|c|c|c|c|c|}
\hline $\begin{array}{c}\text { Height } \\
(\mathrm{m})\end{array}$ & Case 1 & Case 2 & Case 3 & Case 4 & Case 5 & Average \\
\hline$\left[\begin{array}{ll}2.7 & 3.3\end{array}\right]$ & $-17 \%$ & $30 \%$ & $-7.7 \%$ & $-8.5 \%$ & $16 \%$ & $2.3 \%$ \\
\hline$\left[\begin{array}{ll}2.1 & 2.7\end{array}\right]$ & $9.1 \%$ & $5.0 \%$ & $31 \%$ & $7.9 \%$ & $10 \%$ & $6.8 \%$ \\
\hline$\left[\begin{array}{ll}1.5 & 2.1\end{array}\right]$ & $8.6 \%$ & $-28 \%$ & $0.4 \%$ & $21 \%$ & $10 \%$ & $2.9 \%$ \\
\hline$\left[\begin{array}{lll}0.9 & 1.5\end{array}\right]$ & $-0.8 \%$ & $1.3 \%$ & $1.6 \%$ & $-6.6 \%$ & $0.6 \%$ & $0.1 \%$ \\
\hline$\left[\begin{array}{ll}0 & 0.9\end{array}\right]$ & $-6.6 \%$ & $8.5 \%$ & $-2.5 \%$ & $-22 \%$ & $39 \%$ & $3.2 \%$ \\
\hline$\left[\begin{array}{ll}0 & 3.3\end{array}\right]$ & $1.5 \%$ & $-2.6 \%$ & $7.1 \%$ & $0.7 \%$ & $10.8 \%$ & $1.9 \%$ \\
\hline
\end{tabular}

Another important aspect is the procedure to obtain the volume of the tree or, more specifically, the procedure to compute $S_{i j}$. In this work $S_{i j}$ is computed using the rectangular projection of the data points obtained with the LIDAR, as many others authors do $[9,10]$. However, the LIDAR provides a cylindrical projection of the environment and other methods can be used to compute the surface between two measurement points of the laser. 
There are two additional problems not covered in this work: the grass identification and the localization of the center of the tree. The grass identification is very simple because the relative height of the LIDAR is a known parameter and the height of the grass can be deduced directly form some points of the LIDAR slice. Once we have an estimation of its height all laser points under this value can be discarded for the estimation of the volume of the tree. The automatic localization of the center of the tree line is a non trivial problem and very important to obtain a good calibration, and a good estimation of the foliar surface of each side of the tree line in real-time. This problem will be analyzed in deep in future works.

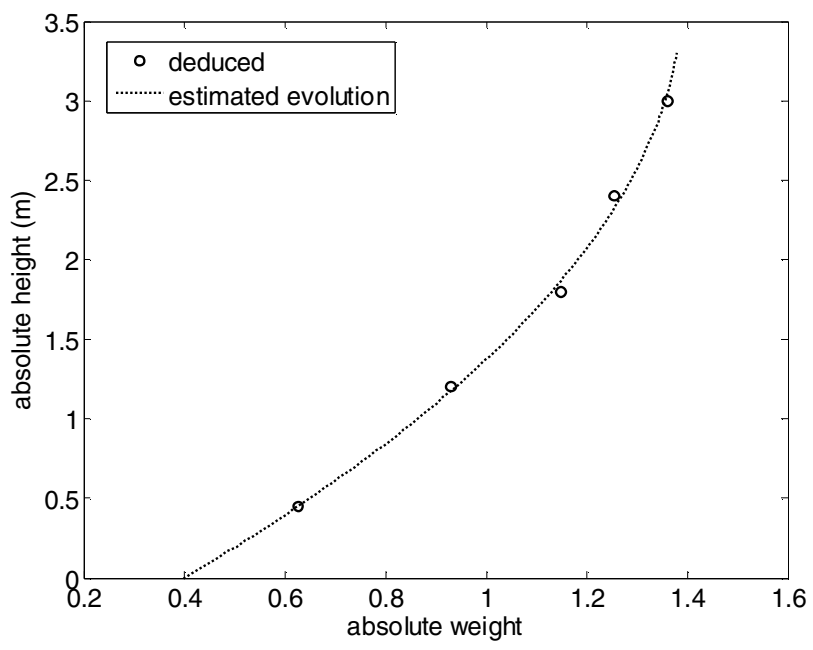

Fig. 11. Values of the weight applied to correct the foliar surface estimation depending on the height.

\section{V.CONCLUSIONS}

In this work a measurement system based on a LIDAR has been proposed and tested for tree foliage estimation. The main advantage of the system is its simplicity, allowing the use of a compact programmable automation controller as a control system instead of a dedicated computer. The foliar surface is estimated through the measurement of the volume that is computed directly form the slices generated by the LIDAR. The main conclusion is that the relation between the volume and the foliar surface can be considered linear in average with an error less than $5 \%$ in the foliage estimation for a complete grove. However, errors up to $93 \%$ can be expected when using the volume to estimate the foliar surface for different heights if no additional calibration is performed. This is a preliminary work and several aspects must be studied in deep as the relation between the volume and the foliar surface, the needed for a specific calibration depending on the species measured, the procedure to compute the volume, the procedure to estimate and compensate the trunk of the trees, and the procedure to detect the center of the trees. However, despite the cited problems, the simplicity of the proposed method will allow height-dose control in pesticide and fertilizer machines with an average error less than $5 \%$ in the foliage estimation for a complete grove, an improvement over other works where only the volume or density are estimated. Acknowledgment is needed.

\section{ACKNOWLEDGMENT}

This work was partially funded by the Spanish Comisión Interministerial de Ciencia y Tecnología, under Agreement No. AGL2002-04260-C04-02.

\section{REFERENCES}

[1] Giles, D.K, M.J. Delwiche, R.B. Dodd (1988). "Electronic measurement of tree canopy volume", Transactions of the ASAE 31(1): 264-272.

[2] Li, B, Whitney, JD, Miller, WM, Wheaton, TA (2001). Ultrasonicbased canopy volume measurements of citrus trees. ASAE Paper No. 021053. St. Joseph, Mich.: ASAE.

[3] Tumbo, S.D., M. Salyani, J.D. Whitney, T.A. Wheaton, and W.M. Miller. (2002). Investigation of laser and ultrasonic ranging sensors for measurements of citrus canopy volume. Applied Engineering in Agriculture. 18: 367-372.

[4] Zaman, Q.U.and M. Salyani. (2004). Effects of foliage density and ground speed on ultrasonic measurement of citrus tree volume. Applied Engineering in Agriculture. 20: 173-178.

[5] Ritchie, J.C., Evans, D.L., Jacobs, D., Everitt, J.H. \& Weltz, M.A (1993). Measuring canopy structure with an airbone laser altimeter. Transaction of the ASAE. : 36, 1235-1238.

[6] Nilsson, M., (1996). Estimation of tree heights and stand. volume using an airborne lidar system. Remote Sensing of. Environment, 56, pp. 1-7.

[7] Walklate P, Cross J, Richardson G, Murray R, Baker D (2002), "Comparison of different spray volume deposition models using LIDAR measurements of apple orchards", In: Biosystems Engineering, 82(3), 253-267.

[8] Tumbo SD, Salyani M, Whitney JD, Wheaton TA, Miller WM. (2002) Investigation of laser and ultrasonic ranging sensors for measurements of citrus canopy volume. In: Applied Engineerig in Agriculture, 18(3), 367-372.

[9] Wei J and Salyani M (2004) Development of a laser scanner for measuring tree canopy characteristics: Phase 1. Prototype development. Transactions of the ASAE, 47(6), 2101-2107.

[10] Wei J and Salyani M (2005) Development of a laser scanner for measuring tree canopy characteristics: Phase 2. Foliage density measurement. Transactions of the ASAE, 48(4), 1595-1601.

[11] Sanz R, Palacín J, Sisó JM, Ribes-Dasi M, Masip J, Arnó J, Llorens J, Vallés J M, Rosell JR. (2004). Advances in the measurement of structural characteristics of plants with a LIDAR scanner. In: Eur AgEng Conference. Leuven. Paper No 277.

[12] LMS 200/211/220/221/291 Laser Measurement Systems: Technical Description. Sick AG, Germany.

[13] Palojarvi P., Maatta K., Kostamovaara J., "Pulsed time-of-flight laser radar module with millimeter-level accuracy using full custom receiver and TDC ASICs", IEEE Transactions on Instrumentation and Measurement, Vol. 51, No. 5, pp. 1102-1108, Oct. 2002.

[14] Nissinen J., Kostamovaara J. (2004), "Wide dynamic range CMOS receivers for a pulsed time-of-flight laser range finder", Proceedings of the IEEE Instrumentation and Measurement Technology Conference, Vol. 2, pp. 1224-1227.

[15] F.M. Turrell, M.J. Garber, W.W. Jones, W.C. Cooper, R.H. Young. "Growth equations and curves for citrus trees. Hilgardia Vol. 39 No. 16, pp. 429-445, 1969. 